\title{
Correction on Drag Coefficient in Simulation of Fast Fluidized Beds
}

\author{
L. M. Zou, Y. C. Guo* \\ Department of Engineering Mechanics, Tsinghua University, Beijing 100084, China \\ Email: guoyc@tsinghua.edu.cn
}

\begin{abstract}
The drag coefficient is of crucial importance for the simulation of the heterogeneous gas-solid flows in fast fluidized system, which is greatly affected by the clustering nature of fast fluidized bed. When simulating fast fluidized system based, the simulated particle should be treated as an element of local cluster. So, the drag coefficient of the single particle approaches to that of some cluster which it belongs to. Then, the characteristic length used in calculating Reynolds number of particle is hydrodynamic equivalent cluster diameter [1]. In this paper, Lagrangian approach was used to predict the motion of particles. The collisions between particles were modeled by means of direct simulation Monte Carlo method.
\end{abstract}

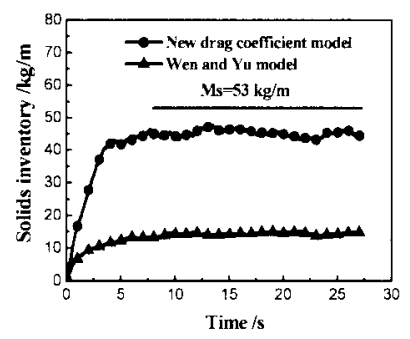

Figure 1: Solids mass inventory in the bed

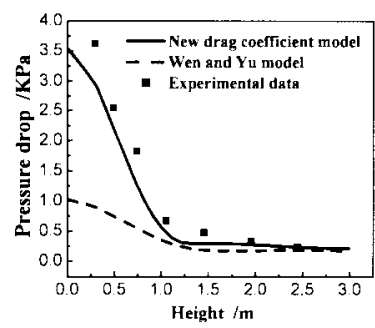

Figure 2: Pressure drop along the height of the bed

Figure 1 shows the changing process of solids inventory in the fast fluidized bed predicted by both of Wen and Yu model [2] and the new drag coefficient model. When using the new drag coefficient model with considering cluster effect, predicted solid mass inventory is about $45 \mathrm{~kg} / \mathrm{m}$ after $5.0 \mathrm{~s}$. However, when using the conventional drag model, predicted solid mass inventory is only about $12 \mathrm{~kg} / \mathrm{m}$ which is much lower than the experimental data of $53 \mathrm{~kg} / \mathrm{m}$. Figure 2 shows the pressure drop along the height of the fast fluidized bed. Compared with the experimental data, predicted results given by the new drag coefficient model are more reasonable than that by Wen and Yu model, this indicates that the new drag coefficient model is capable of describing the actual status of gas-solid fast fluidized beds.

\section{Acknowledgements}

This work was supported by the National Natural Science Foundation of China (No.50376028), and jointly by NSFC and PetroChina (No.20490200).

\section{REFERENCES}

1. Xu GW, Kato K. Hydrodynamic equivalent diameter for clusters in heterogeneous gas-solid flow. Chemical Engineering Science, 1999;54:1837-1847

2. Wen CY, Yu YH. Mechanics of fluidization. Aiche Symp., 1966;62:100-111 